\title{
Efficacy of long-term ultrasonography in follow-up of patients with papillary thyroid carcinoma: a case of neck metastasis developed 19 years following primary treatment
}

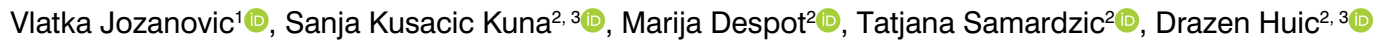 \\ 'Department of Nuclear Medicine, Clinical Hospital Centre Dubrava, Croatia \\ ${ }^{2}$ Clinical Department of Nuclear Medicine and Radiation Protection, University Hospital Centre Zagreb, Croatia \\ ${ }^{3}$ The University of Zagreb School of Medicine, Zagreb, Croatia
}

[Received 01 IV 2021; Accepted 07 VII 2021]

\begin{abstract}
The cases of relapse in papillary thyroid cancer patients who were initially considered low-risk and for many years were without signs of the disease are extremely rare, but exist. This is supported by the clinical case of a patient who underwent a total thyroidectomy due to papillary thyroid cancer and 19 years later metastasis with extracapsular spreading in a presumed thyroid place was revealed. Due to such cases, the importance of long-term ultrasound monitoring is emphasized.
\end{abstract}

KEY words: papillary thyroid carcinoma; neck metastases; follow-up

Nucl Med Rev 2022; 25, 1: 62-63

\section{Introduction}

The metastases of papillary thyroid cancer usually occur during the first few years after the initial procedure and generally develop in patients with a higher risk of persistent or recurrent disease such as the presence of local invasion and metastases. Ultrasound is the best method for detection of those lesions in the neck, it provides reliable information regarding the lesion size, shape, internal architecture, and vascularity and is recommended during regular intervals throughout life. The aim of this report is to present the occurrence of cystic metastasis in the neck 19 years after total thyroidectomy in a patient who was considered low-risk and did not have any recurrence of the disease at regular follow-up for many years.

Correspondence to: Sanja Kusacic Kuna, Clinical Department of Nuclear Medicine and Radiation Protection, University Hospital Centre Zagreb, Kispaticeva 12, 10000 Zagreb, Croatia, tel.: 0038598 238315, fax number: +38512376 040

e-mail: sanja.kusacickuna@gmail.com

\section{Case report}

A 27-years old female patient underwent total thyroidectomy and radioiodine ablation in 2000 due to accidental ultrasound finding of small papillary thyroid cancer in the left lobe that was confirmed cytologically and then histologically. During regular follow-up, the patient was free of disease with a normal thyroglobulin level and completely normal neck ultrasound. But, at regularly scheduled examination at the end of 2019 ultrasound revealed a newly formed hypoechoic, avascular cystic formation in the right thyroid bed measuring $0.7 \times 0.8 \times 0.7 \mathrm{~cm}$ (Fig. 1). Ultrasound-guided FNAB indicated a suspicious cystic change with atypia of thyrocytes and elevated thyroglobulin level in the punctate content that amounted to $71.50 \mu \mathrm{g} / \mathrm{L}$. The patient was referred for paratracheal dissection during which the formation was extirpated. The result of pathohistological analysis proved it was a metastasis of papillary thyroid carcinoma, in a nodule of $0.6 \mathrm{~cm}$ in diameter with extracapsular spreading that penetrates the capsule of the affected lymph node. Due to the extracapsular spreading patient was treated with a therapeutic dose of $3700 \mathrm{MBq}(100 \mathrm{mCi})$ of iodine-I31. Post-therapeutic

This article is available in open access under Creative Common Attribution-Non-Commercial-No Derivatives 4.0 International (CC BY-NC-ND 4.0) license, allowing to download articles and share them with others as long as they credit the authors and the publisher, but without permission to change them in any way or use them commercially. 

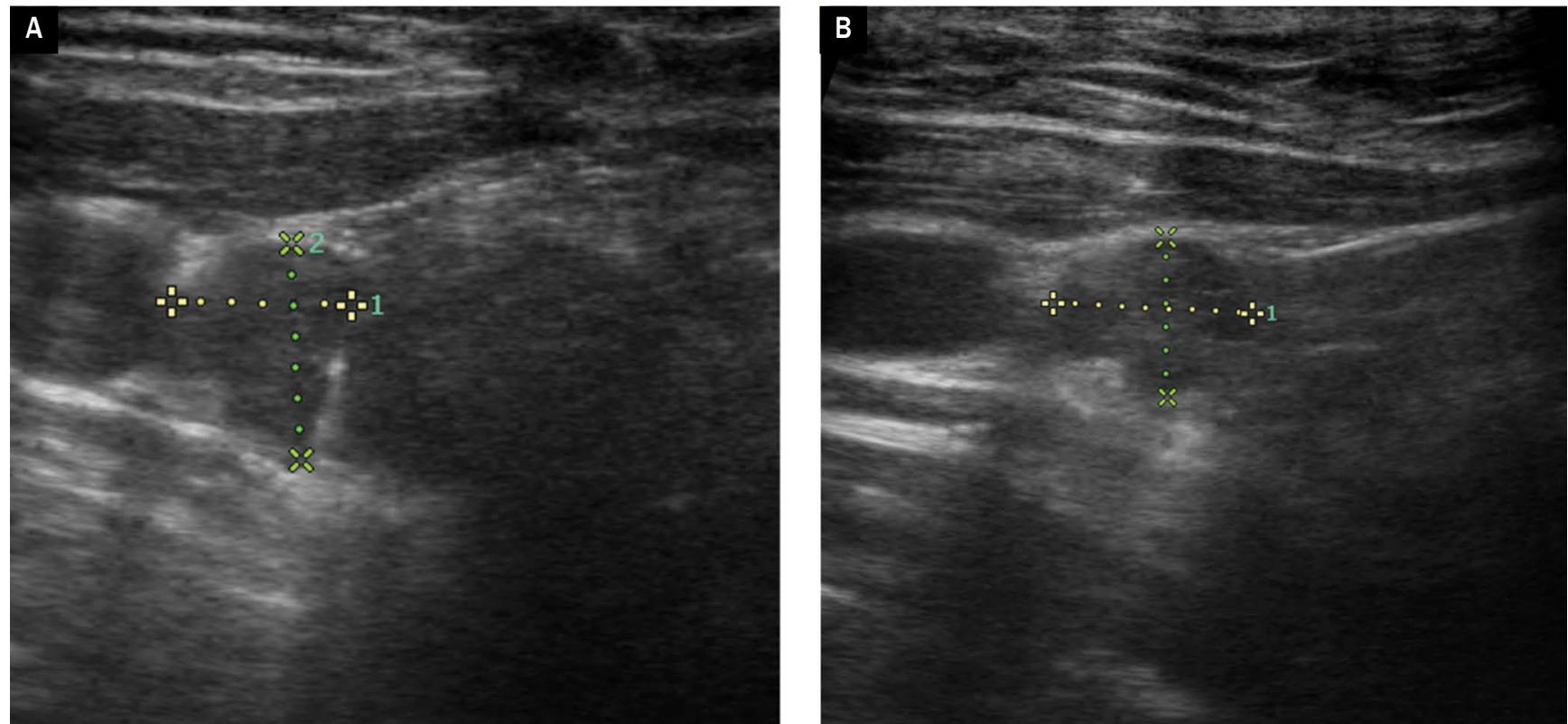

Figure 1A-B. Echographic presentation of surgically resected and histologically proven cystic metastasis in right thyroid bed

whole-body scan verified pathological accumulation of low intensity in the medial line of the neck. Postoperative ultrasound of the neck did not reveal any residual formation in the thyroid bed as well as elsewhere in the neck, and thyroglobulin level was within the reference ranges expected in thyroidectomized patients $(<0.30 \mu \mathrm{g} / \mathrm{L})$. At the regular follow-up performed ten months after the surgery, the patient had no signs of recurrence of the disease. This case of metastasis occurred almost 20 years after initial treatment reports in a favor of lifelong ultrasonographic follow-up of patients with papillary thyroid cancer.

\section{Conflict od interest}

The authors declare that they have no conflict of interest. 